\title{
Peripheral Arterial Disorder
}

National Cancer Institute

\section{Source}

National Cancer Institute. Peripheral Arterial Disorder. NCI Thesaurus. Code C84496.

A disorder of the arteries supplying the upper and lower extremity and the visceral

organs. This includes the mesenteric arteries, the renal arteries and the aorta and

excludes cerebrovascular arterial disease. Patients experience cramping and pain usually

in the calves and thighs while walking. The symptoms subside with rest. 